\title{
On upwind methods for parabolic finite elements in incompressible flows
}

\author{
Dena Hendriana and Klaus-Jürgen Bathe* \\ Department of Mechanical Engineering, Massachusetts Institute of Technology, 77 Massachusetts Avenue, \\ Cambridge, MA 02139, U.S.A.
}

\begin{abstract}
SUMMARY
We study the performance of various upwind techniques implemented in parabolic finite element discretizations for incompressible high Reynolds number flow. The characteristics of an 'ideal' upwind procedure are first discussed. Then the streamline upwind Petrov/Galerkin method, a simplified version thereof, the Galerkin least squares technique and a high-order derivative artificial diffusion method are evaluated on test problems. We conclude that none of the methods displays the desired solution characteristics. There is still need for the development of a reliable and efficient upwind method with characteristics close to those of the 'ideal' procedure. Copyright (C) 2000 John Wiley \& Sons, Ltd.
\end{abstract}

KEY WORDS: incompressible fluid; upwinding; parabolic elements

\section{INTRODUCTION}

In the finite element method using the standard Galerkin procedure for incompressible flows, there are two major sources of numerical instability. The first source of instability is due to an inappropriate combination of element interpolation functions for the velocity and pressure. This instability of the formulation is observed even in very low Reynolds number flows. The other source of instability is due to the presence of the convective term and is observed when the convective term is dominant.

To remedy the first source of numerical instability, the theory of the inf-sup condition for incompressible media is available, see, e.g. $[1,2]$. It is shown that the finite element method is optimally convergent if an appropriate combination of finite element spaces for velocity and pressure are used to satisfy the inf-sup condition for incompressible media. This condition limits the combinations of appropriate velocity and pressure interpolation functions. Some methods have also been proposed to stabilize the finite element formulation while still using equal-order of interpolations for the velocity and pressure, see, e.g. [1,3-6]. In these methods, a numerical stabilization term is introduced to 'damp' the pressure oscillations.

To remedy the second source of numerical instability, upwind methods are employed. Various upwind techniques have been proposed and are employed in finite element solutions, including

\footnotetext{
* Correspondence to: Klaus-Jürgen Bathe, Department of Mechanical Engineering, Massachusetts Institute of Technology, 77 Massachusetts Avenue, Cambridge, MA 02139, U.S.A. E-mail: kjb@mit.edu
}

CCC 0029-5981/2000/020317-24\$17.50

Received 12 November 1998

Copyright (c) 2000 John Wiley \& Sons, Ltd. 
the Streamline Upwind/Petrov Galerkin (SUPG) method [7, 8], the Galerkin Least Squares (GLS) method [9], upwinding based upon bubble functions [3, 10, 11], the Taylor-Galerkin based method $[12,13]$ and the High-order Derivative Artificial Diffusion (HDAD) method [14]. A few comparisons of some of these techniques are available [15], but there is still little understanding as to how these methods perform comparatively when used in a broad spectrum of solutions.

In solving a high Reynolds number problem, an 'ideal' solution scheme would have the following properties.

1. Property of discretization errors: uniform and optimal convergence of the finite element solutions to the solution of the mathematical model, and

2. Property of solution of finite element equations: fast convergence in the iterations to solve the algebraic finite element equations for any mesh and up to very high Reynolds number flows.

The second property is clearly necessary in order to have an effective solution scheme. Note the emphasis on obtaining the finite element solution for any-of course, reasonable-mesh and very high Reynolds number flows.

The first property entails a number of requirements, namely

(1) For any mesh, the method should give a reasonable solution.

(2) The method should give the highest possible (optimal) convergence in discretization errors.

(3) The method should not be 'too sensitive' to the mesh used.

(4) For any mesh, an error indicator should be available to evaluate the quality of the solution.

(5) If the error indicator indicates too large an error, refining the mesh should result into a good solution with an acceptable error.

(6) The method should converge to the 'exact' solution of the mathematical model.

Considering these requirements, a reasonable solution means that the solution does not contradict intuition or physical reasoning; for example the direction of flow should be intuitively correct. The solutions should have no oscillations and as the mesh is refined, the overall flow field should continuously change to approach the 'exact' solution of the mathematical model. Hence, denoting the solution obtained using a given mesh by $\mathbf{U}($ mesh), we want to have

$$
\mathbf{U}(\text { coarse mesh }) \subset \mathbf{U}(\text { fine mesh }) \subset \mathbf{U}(\text { finer mesh }) \subset \ldots
$$

The mathematical model we consider in this study is governed by the Navier-Stokes equations. Hence, at the scale considered, laminar flow is assumed although high Reynolds number conditions are specified. The upwind finite element solution procedure should be able to solve for the laminar flow at high Reynolds number for at least two reasons. First, the laminar flow assumption is generally used in the numerical solution as an initial approximation. Therefore, if the solution cannot be obtained with this approximation, it is difficult to continue the iterative solution introducing appropriate turbulence models. Second, at high Reynolds number, the flow is frequently only turbulent in certain areas, and is still laminar in the other parts of the fluid domain.

When the mesh is very coarse, the method should be able to obtain 'a reasonable' solution even though the actual physical flow might contain instabilities. With a coarse mesh, the solution will not be able to represent all the physical phenomena, such as boundary layers, backflows, or instabilities, but as the mesh is refined, increasingly more physical phenomena should be revealed in the solution, while the overall flow should be similar to the coarse mesh solution. Eventually, when the mesh is fine enough, the method should also be able to solve for an unstable behaviour of the flow in which case a transient analysis need be used. 
For example, let us consider the driven flow cavity problem, see Figure 1 . The domain is discretized initially into a mesh of $10 \times 10$ elements. The method should be able to solve the problem when the Reynolds number is low, such as 10 or 100 , without too much difficulty. When the Reynolds number is greater than 1000 , and then much greater than 1000 , the iteration might converge more slowly but an ideal method would still obtain a physically reasonable solution. Of course, using the coarse mesh, boundary layers and circulation flows near the corners will not be revealed, but as the mesh is refined, these physical phenomena will be presented.

Ideally, the method should be able to solve any Navier-Stokes flow problem as described above up to a Reynolds number $\sim 10^{7}$, because many engineering problems are defined at such high Reynolds numbers.

The objective of this paper is to test some existing upwind methods for parabolic finite elements and determine whether the methods satisfy the requirements of the 'ideal' scheme discussed above. Simple Galerkin discretizations using parabolic elements are generally more stable than using linear elements; however, upwinding is still needed to solve high Reynolds number flows. The methods that we consider here are the high-order derivative artificial diffusion method which is similar to upwind methods used in finite difference procedures, the Streamline Upwind Petrov/Galerkin (SUPG) method [7,8], a simplification thereof, and the Galerkin Least Squares (GLS) technique [9]. We use four test problems to measure the performance of the methods. Of course, our evaluation is by no means all encompassing in that, for example, we only consider four upwinding procedures, we only solve a few test problems, we do not measure the solution errors, we do not use specially aligned meshes to minimize these errors, and we solve the problems only with parabolic elements. However, although these shortcomings are severe, we believe that the study is valuable in contributing to the comparative evaluation of the existing upwind methods, and might lead towards ideas to improve the solution schemes.

\section{GOVERNING EQUATIONS}

Let $\mathrm{Vol}$ and $(0, T)$ be the spatial and temporal domains, and let $\mathbf{x} \in \mathrm{Vol}$ and $t \in[0, T]$ represent the associated co-ordinates. Using a Cartesian co-ordinate system and indicial notation, the NavierStokes equations for incompressible flow can be written as

$$
\begin{aligned}
v_{i, i}=0 & \text { in } \mathrm{Vol} \times(0, T) \\
\rho v_{i, t}+\rho v_{i, j} v_{j}+p_{, i}-\mu\left(v_{i, j}+v_{j, i}\right)_{, j}-f_{i}^{\mathrm{B}}=0 & \text { in } \mathrm{Vol} \times(0, T)
\end{aligned}
$$

where $v_{i}, \rho, p, \mu$, and $f_{i}^{\mathrm{B}}$ are the velocity in the $x_{i}$-direction, fluid density, pressure, fluid viscosity, and body force per unit volume in the $x_{i}$-direction, respectively, and ()$_{, t},()_{, i}$ denote partial differentiations with respect to time and $x_{i}$-coordinate. The Dirichlet and Neumann type boundary conditions are imposed at different segments of the boundary $S$

$$
\begin{aligned}
v_{i}=g_{i} & \text { on } S_{u} \\
\tau_{i j} n_{j}=h_{i} & \text { on } S_{f}
\end{aligned}
$$

where $g_{i}, h_{i}$ are given functions; $\tau_{i j}$ is the stress component; $n_{j}$ is the component of the boundary unit normal vector and $S_{u}, S_{f}$ are complementary subsets of $S$. Note that we do not consider the energy equation because the finite element solution of the above equations will already reveal sufficiently the basic characteristics of the solution schemes. 

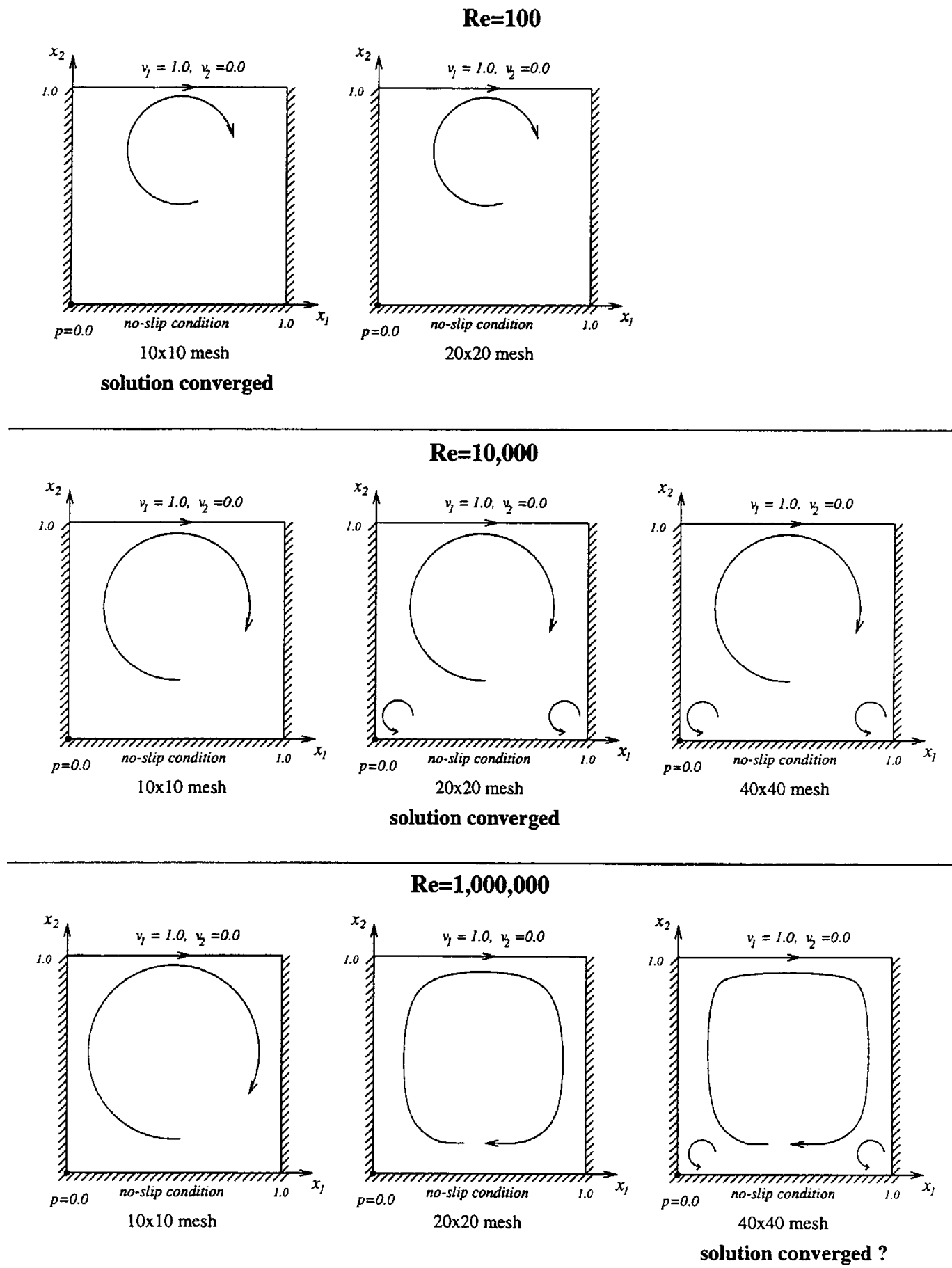

Figure 1. Schematics of solutions of driven flow cavity problem 


\section{FINITE ELEMENT DISCRETIZATIONS}

We want to evaluate the performance of upwind methods when using parabolic element discretizations. For incompressible flow at low Reynolds number the most effective nine-node elements are the 9/3 (linear pressure interpolation) element and the 9/4-c (bilinear continuous pressure interpolation) element. These discretizations contain the highest possible order of pressure interpolation while satisfying the inf-sup condition for incompressible analysis (without use of a numerical factor as employed in stabilized finite element discretizations), and are therefore excellent candidates for high Reynolds number flow solutions. We use the 9/4-c element for the solution of high Reynolds number flow with the SUPG, simplified SUPG and high-order derivative artificial diffusion upwind methods embedded in the discretizations. On the other hand, for the Galerkin least squares method we use the usual approach of equal-order interpolations and numerically stabilizing the velocity and pressure components [1]. Hence, we use the nine-node element with all nodes employed to interpolate the velocity and pressure variables.

\subsection{High-order Derivative Artificial Diffusion (HDAD) method}

The high-order derivative artificial diffusion method is considered because the method is very simple and computationally efficient. The method for incompressible flows is an extention of the technique used for compressible flows [14]. In the solution, the two-dimensional quadrilateral 9/4-c element is employed to discretize the domain.

The solution and weighting functional linear spaces ${ }^{\dagger}$ are

$$
\begin{aligned}
V_{h}= & \left\{\mathbf{v}_{h} \mid \mathbf{v}_{h} \in L^{2}(\mathrm{Vol}) ; \frac{\partial\left(v_{h}\right)_{i}}{\partial x_{j}} \in L^{2}(\mathrm{Vol}), i=1,2,3 ; j=1,2 ;\left(v_{h}\right)_{1} \in Q_{1}\left(\mathrm{Vol}^{(m)}\right),\right. \\
& \left.\left(v_{h}\right)_{2,3} \in Q_{2}\left(\mathrm{Vol}^{(m)}\right) ;\left.\left(v_{h}\right)_{i}\right|_{S_{u}}=g_{i}(t), i=2,3\right\} \\
W_{h}= & \left\{\mathbf{w}_{h} \mid \mathbf{w}_{h} \in L^{2}(\mathrm{Vol}) ; \frac{\partial\left(w_{h}\right)_{i}}{\partial x_{j}} \in L^{2}(\mathrm{Vol}), i=1,2,3 ; j=1,2 ;\left(w_{h}\right)_{1} \in Q_{1}\left(\mathrm{Vol}^{(m)}\right),\right. \\
& \left.\left(w_{h}\right)_{2,3} \in Q_{2}\left(\mathrm{Vol}^{(m)}\right) ;\left.\left(w_{h}\right)_{i}\right|_{S_{u}}=0, i=2,3\right\}
\end{aligned}
$$

where, as usual, $L^{2}(\mathrm{Vol})$ is the space of square integrable functions in the volume, and $Q_{1}\left(\mathrm{Vol}^{(m)}\right)$ and $Q_{2}\left(\mathrm{Vol}^{(m)}\right)$ denote the bilinear and biquadratic functions in the reference element $m$.

The finite element formulation for the incompressible flow using the high-order derivative artificial diffusion method is:

Find $\left(p, v_{1}, v_{2}\right) \in V_{h}$ such that for all $\left(\bar{p}, \bar{v}_{1}, \bar{v}_{2}\right) \in W_{h}$

$$
\begin{gathered}
\int_{\mathrm{Vol}} \bar{p} v_{i, i} \mathrm{dVol}=0 \\
\int_{\mathrm{Vol}}\left\{\bar{v}_{i}\left(\rho v_{i, t}+\rho v_{i, j} v_{j}\right)-\bar{v}_{i, i} p+\bar{v}_{i, j} \mu\left(v_{i, j}+v_{j, i}\right)\right\} \mathrm{dVol} \\
+\sum_{m} \int_{\mathrm{Vol}^{(m)}} \bar{v}_{i, j j} \tau_{j} \rho\left|v_{j}\right| v_{i, j j} \mathrm{dVol}^{(m)}=\int_{\mathrm{Vol}} \bar{v}_{i} f_{i}^{\mathrm{B}} \mathrm{dVol}+\int_{S_{f}} \bar{v}_{i}^{S} f_{i}^{S} \mathrm{~d} S
\end{gathered}
$$

\footnotetext{
$\dagger$ Actually, to be precise, $V_{h}$ is not a linear space, but an affine manifold that can be thought of as obtained by translating the linear space $W_{h}$
} 
Regarding this equation, the boundary traction term in the $x_{i}$-direction is defined as

$$
f_{i}^{S}=\left\{-p \delta_{i j}+\mu\left(v_{i, j}+v_{j, i}\right)\right\} n_{j}
$$

where $n_{j}$ is the $x_{j}$-direction cosine of the unit (pointed outward) boundary normal vector. $\delta_{i j}$ is the Kronecker delta (i.e. $\delta_{i j}=1$ for $i=j$, and $\delta_{i j}=0$ for $i \neq j$ ).

The value of $\tau_{j}$ is defined as

$$
\tau_{j}=\frac{1}{9}\left(\left|\frac{\partial x_{j}}{\partial \mathbf{r}}\right|\right)^{3}
$$

where $\mathbf{r}$ denotes the co-ordinates in the natural co-ordinate system of the element. The characteristic length is defined as

$$
\left|\frac{\partial x_{j}}{\partial \mathbf{r}}\right|=\sqrt{\left(\frac{\partial x_{j}}{\partial r_{1}}\right)^{2}+\left(\frac{\partial x_{j}}{\partial r_{2}}\right)^{2}}
$$

The factor $\frac{1}{9}$ in the $\tau_{j}$ definition is used to obtain full upwinding corresponding to the outer nodes of the element in one-dimensional flow conditions.

We note that the form of upwinding used in equation (3) is similar to high-order upwinding in finite difference methods. The upwinding is proportional to the second derivatives of the velocity components, and is therefore of higher order, but in the form of equation (3), the amount of upwinding is dependent on the directions of the co-ordinate axes. Our numerical experimentation showed that this deficiency appears to be not a serious drawback. However, other forms of HDAD upwinding can of course be designed, including a scheme that would only apply artificial diffusion in the streamline direction (and perhaps a controlled amount in the cross-direction).

\subsection{Streamline Upwind/Petrov-Galerkin (SUPG) method}

The SUPG method was originally proposed for the bilinear element [7]. We use the method here for the quadratic 9/4-c element. Some authors extended the SUPG technique for use with a quadratic element by using different definitions of the SUPG parameter $\tau$ for corner, mid-face and centre nodes of the element $[12,16]$. However, this approach leads to a complicated formulation.

The finite element formulation that we use with the SUPG upwinding is:

Find $\left(p, v_{1}, v_{2}\right) \in V_{h}$ such that for all $\left(\bar{p}, \bar{v}_{1}, \bar{v}_{2}\right) \in W_{h}$

$$
\begin{gathered}
\int_{\mathrm{Vol}} \bar{p} v_{i, i} \mathrm{dVol}=0 \\
\int_{\mathrm{Vol}}\left\{\bar{v}_{i}\left(\rho v_{i, t}+\rho v_{i, j} v_{j}\right)-\bar{v}_{i, i} p+\bar{v}_{i, j} \mu\left(v_{i, j}+v_{j, i}\right)\right\} \mathrm{dVol} \\
+\sum_{m} \int_{\mathrm{Vol}^{(m)}} \bar{v}_{i, k} v_{k} \tau\left\{\rho v_{i, t}+\rho v_{i, j} v_{j}+p_{, i}-\mu\left(v_{i, j}+v_{j, i}\right)_{, j}-f_{i}^{\mathrm{B}}\right\} \mathrm{dVol}^{(m)} \\
=\int_{\mathrm{Vol}} \bar{v}_{i} f_{i}^{\mathrm{B}} \mathrm{dVol}+\int_{S_{f}} \bar{v}_{i}^{S} f_{i}^{S} \mathrm{~d} S
\end{gathered}
$$

The value of $\tau$ is defined as

$$
\tau=h \xi\left(R e^{e}\right) / 2 V
$$




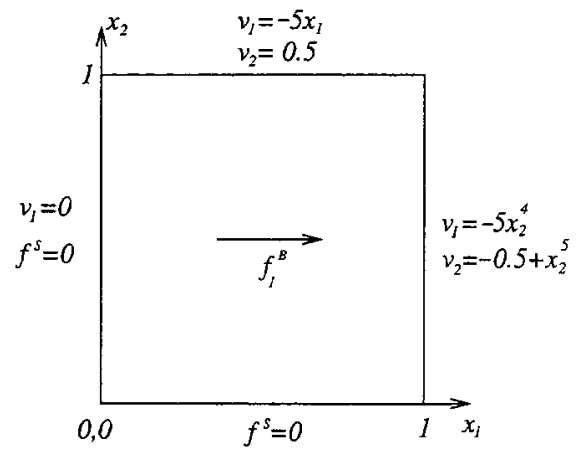

Figure 2. Impinging fluid flow over a slip-wall problem

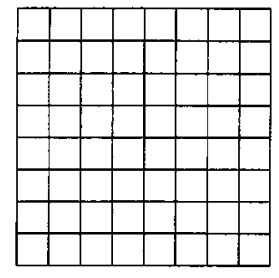

(a)

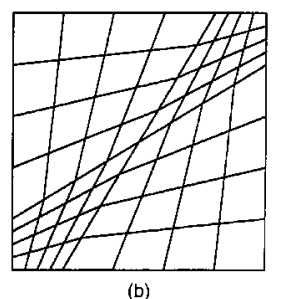

(b)

Figure 3. Meshes used for the impinging fluid flow over a slip-wall problem: (a) uniform; (b) distorted

where $h$ is the characteristic length of the element, and

$$
\begin{aligned}
R e^{e} & =\rho V h / \mu \\
V & =\sqrt{v_{1}^{2}+v_{2}^{2}} \\
\xi\left(R e^{e}\right) & = \begin{cases}R e^{e} / 3, & R e^{e} \leqslant 3 \\
1, & R e^{e}>3\end{cases}
\end{aligned}
$$

\subsection{Simplified SUPG (S-SUPG) procedure}

In this solution we also use the 9/4-c element. The finite element formulation for the incompressible flow using the simplified SUPG technique is:

Find $\left(p, v_{1}, v_{2}\right) \in V_{h}$ such that for all $\left(\bar{p}, \bar{v}_{1}, \bar{v}_{2}\right) \in W_{h}$

$$
\begin{gathered}
\int_{\mathrm{Vol}} \bar{p} v_{i, i} \mathrm{dVol}=0 \\
\int_{\mathrm{Vol}}\left\{\bar{v}_{i}\left(\rho v_{i, t}+\rho v_{i, j} v_{j}\right)-\bar{v}_{i, i} p+\bar{v}_{i, j} \mu\left(v_{i, j}+v_{j, i}\right)\right\} \mathrm{dVol} \\
+\sum_{m} \int_{\mathrm{Vol}^{(m)}} \bar{v}_{i, k} v_{k} \tau\left\{\rho v_{i, j} v_{j}-f_{i}^{\mathrm{B}}\right\} \mathrm{dVol}^{(m)}=\int_{\mathrm{Vol}} \bar{v}_{i} f_{i}^{\mathrm{B}} \mathrm{dVol}+\int_{S_{f}} \bar{v}_{i}^{S_{f}} f_{i}^{S} \mathrm{~d} S
\end{gathered}
$$


The definition of $\tau$ is as for the SUPG method. The reasoning for using this method is that the convective term in the stabilization is dominant and the pressure and diffusive terms might not be needed. Also, the transient term should not be needed since the upwinding is used to stabilize the spatial solution variation.

\subsection{Galerkin Least Squares (GLS) method}

In this method, the 9-node (9/9-c) element is employed to discretize the domain with the usual 9-node interpolation of the velocity and the same 9-node interpolation for the pressure. The solution and weighting functional linear spaces ${ }^{\ddagger}$ are

$$
\begin{aligned}
V_{h}= & \left\{\mathbf{v}_{h} \mid \mathbf{v}_{h} \in L^{2}(\mathrm{Vol}) ; \frac{\partial\left(v_{h}\right)_{i}}{\partial x_{j}} \in L^{2}(\mathrm{Vol}), i=1,2,3 ; j=1,2 ;\right. \\
& \left.\left(v_{h}\right)_{1,2,3} \in Q_{2}\left(\mathrm{Vol}^{(m)}\right) ;\left.\left(v_{h}\right)_{i}\right|_{S_{u}}=g_{i}(t), i=2,3\right\} \\
W_{h}= & \left\{\mathbf{w}_{h} \mid \mathbf{w}_{h} \in L^{2}(\mathrm{Vol}) ; \frac{\partial\left(w_{h}\right)_{i}}{\partial x_{j}} \in L^{2}(\mathrm{Vol}), i=1,2,3 ; j=1,2 ;\right. \\
& \left.\left(w_{h}\right)_{1,2,3} \in Q_{2}\left(\mathrm{Vol}^{(m)}\right) ;\left.\left(w_{h}\right)_{i}\right|_{S_{u}}=0, i=2,3\right\}
\end{aligned}
$$

The finite element formulation for the incompressible flow using the Galerkin least squares method is:

Find $\left(p, v_{1}, v_{2}\right) \in V_{h}$ such that for all $\left(\bar{p}, \bar{v}_{1}, \bar{v}_{2}\right) \in W_{h}$

$$
\begin{gathered}
\int_{\mathrm{Vol}} \bar{p} v_{i, i} \mathrm{dVol}+\sum_{m} \int_{\mathrm{Vol}^{(m)}} \bar{p}_{, i} \tau\left\{\rho v_{i, t}+\rho v_{i, j} v_{j}+p_{, i}\right. \\
\left.-\mu\left(v_{i, j}+v_{j, i}\right)_{, j}-f_{i}^{\mathrm{B}}\right\} \mathrm{dVol}^{(m)}=0 \\
\int_{\mathrm{Vol}}\left\{\bar{v}_{i}\left(\rho v_{i, t}+\rho v_{i, j} v_{j}\right)-\bar{v}_{i, i} p+\bar{v}_{i, j} \mu\left(v_{i, j}+v_{j, i}\right)\right\} \mathrm{dVol} \\
+\sum_{m} \int_{\mathrm{Vol}^{(m)}}\left\{\rho \bar{v}_{i, t}+\rho \bar{v}_{i, k} v_{k}-\mu\left(\bar{v}_{i, k}+\bar{v}_{k, i}\right)_{, k}\right\} \tau\left\{\rho v_{i, t}+\rho v_{i, j} v_{j}+p_{, i}\right. \\
\left.-\mu\left(v_{i, j}+v_{j, i}\right)_{, j}-f_{i}^{\mathrm{B}}\right\} \mathrm{dVol}^{(m)}=\int_{\mathrm{Vol}} \bar{v}_{i} f_{i}^{\mathrm{B}} \mathrm{dVol}+\int_{S_{f}} \bar{v}_{i}^{S} f_{i}^{S} \mathrm{~d} S
\end{gathered}
$$

The value of $\tau$ was defined in References [9, 17]

$$
\tau=h \xi\left(R e^{e}\right) / 2 \rho V
$$

where

$$
\begin{aligned}
R e^{e} & =m_{h} \rho V h / 4 \mu \\
V & =\sqrt{v_{1}^{2}+v_{2}^{2}} \\
\xi\left(R e^{e}\right) & = \begin{cases}R e^{e}, & R e^{e}<1 \\
1, & R e^{e} \geqslant 1\end{cases}
\end{aligned}
$$

$\ddagger$ See footnote 1 


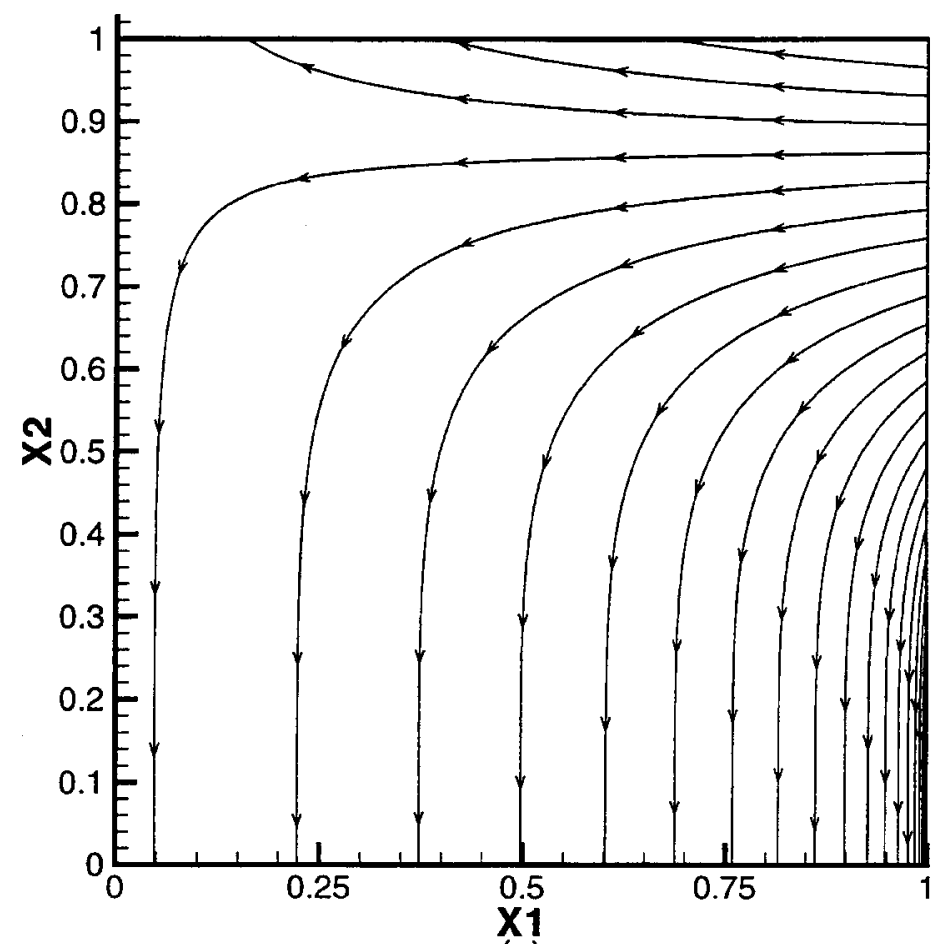

(a)

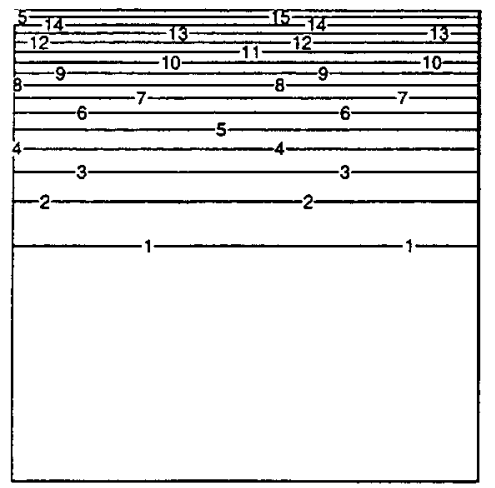

(b)

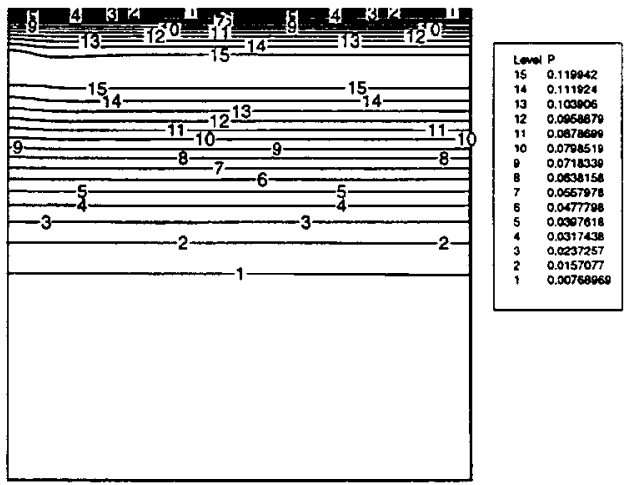

(c)

Figure 4. Solution of impinging fluid flow over a slip-wall using the 9/9-c element with the GLS method: (a) streamlines; (b) pressure in case 1; (c) pressure in case 2

and $m_{h}=1 / 12$ for quadratic elements, see [15]. Hence, with this definition, maximum upwinding is reached when $\rho V h / \mu=36$, whereas in the SUPG method when $\rho V h / \mu=3$. 


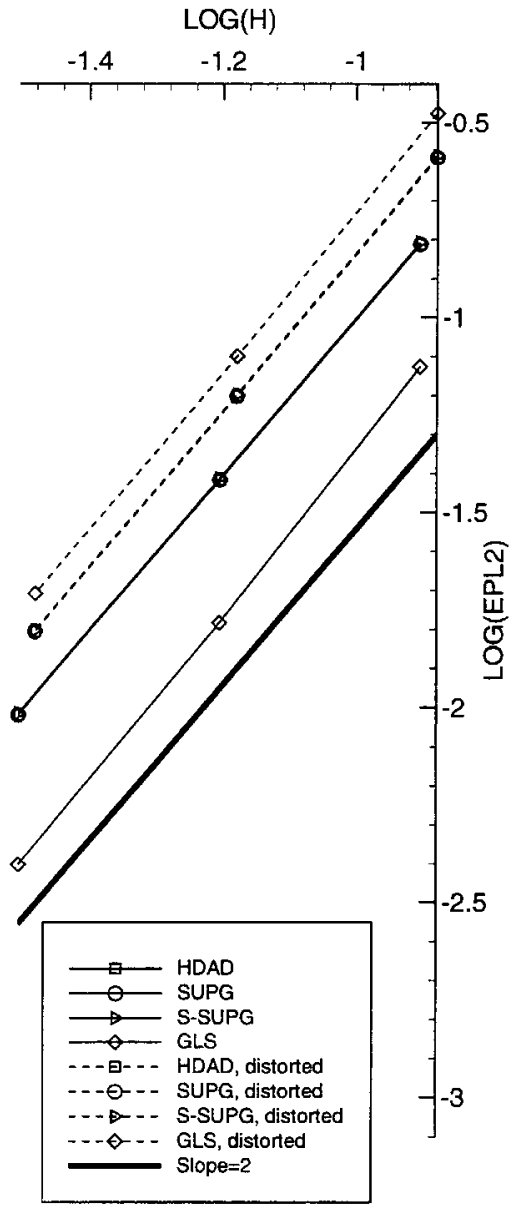

(a)

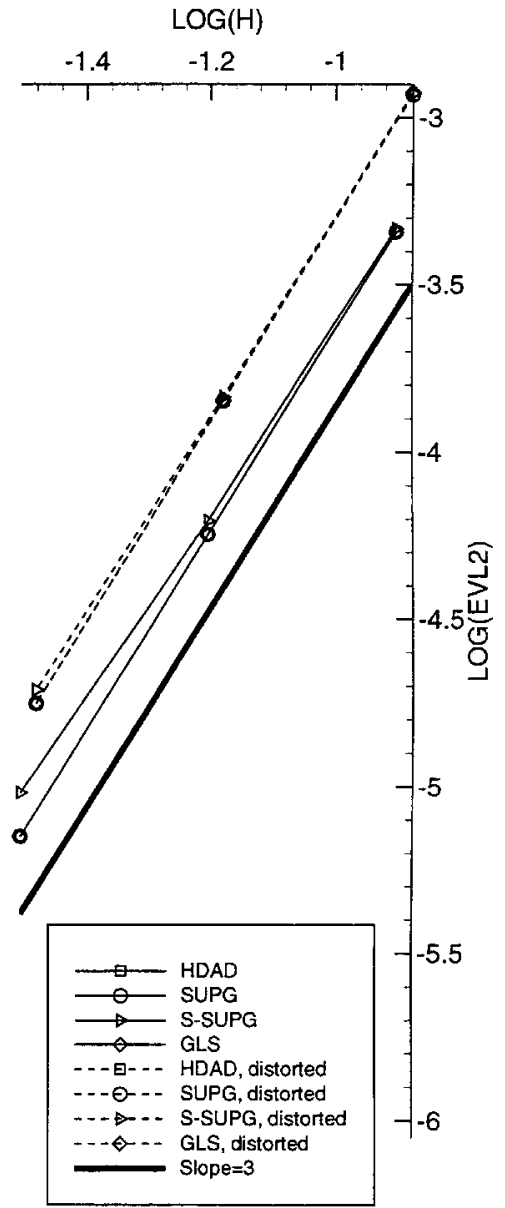

(b)

Figure 5. Convergence curves for the impinging fluid flow over a slip-wall problem, case 1: (a) pressure error in $L^{2}$-norm; (b) velocity error in $L^{2}$-norm

\section{NUMERICAL TESTS}

In this section we study the performance of the discretization and upwind methods summarized in the previous section. First, we apply the methods to the solution of a problem, for which we have the exact analytical solution, and we estimate the convergence rates of the methods. Then we consider three problems to solve high Reynolds number flows. To solve the nonlinear equations, we use the Newton-Raphson method and allow a maximum of 35 iterations to reach the solution. We report the number of iterations required to solve the problems at different Reynolds numbers and report the largest Reynolds number for which the methods yield the solutions.

The problem solutions can of course also be sought using the Galerkin method without upwinding, and depending on the problem, convergence in the solution of the algebraic equations 


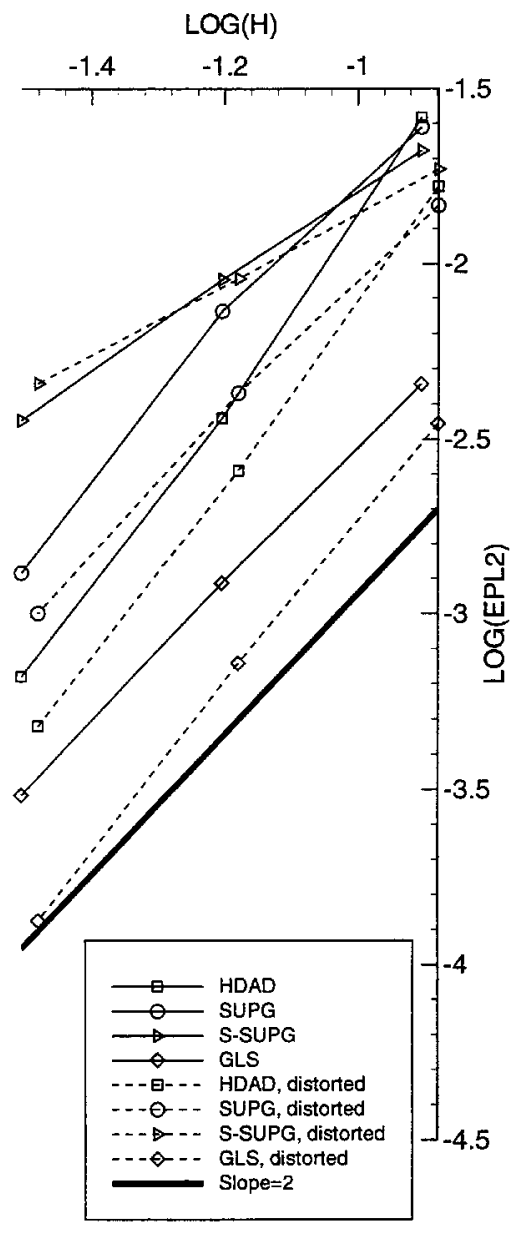

(a)

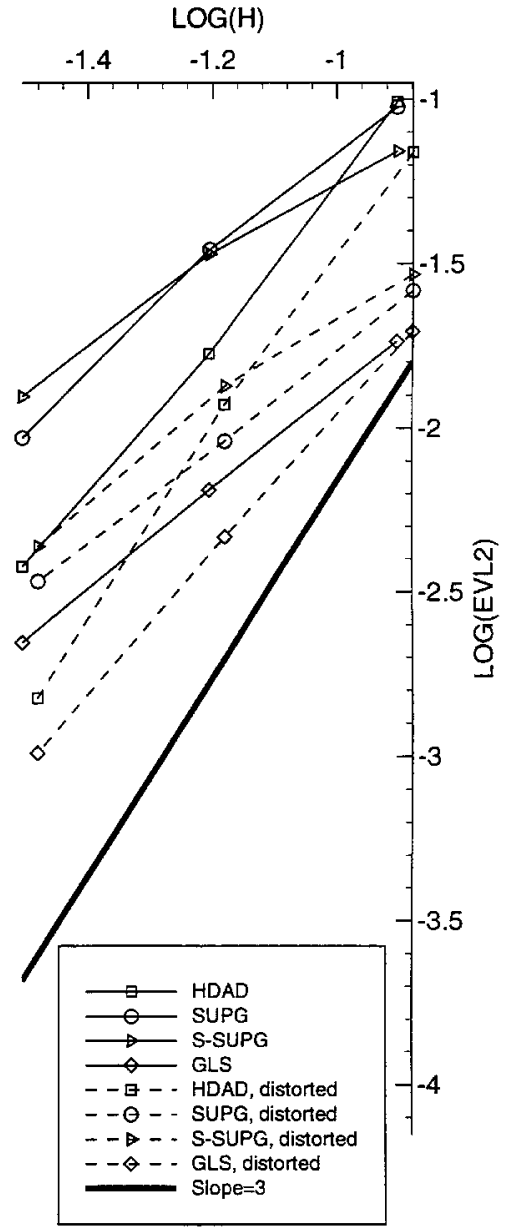

(b)

Figure 6. Convergence curves for the impinging fluid flow over a slip-wall problem, case 2: (a) pressure error in $L^{2}$-norm; (b) velocity error in $L^{2}$-norm

is obtained for some reasonably high Reynolds number flows. However, the converged solutions show oscillations and we do not include the results in this paper.

\subsection{Convergence study problem}

We consider the problem described in Figure 2. The problem consists of a jet impinging upon a wall with a controlled body force. The fluid is assumed to slip on the wall. The body force functions are

$$
\begin{aligned}
& f_{1}^{\mathrm{B}}=5 x_{1} x_{2}^{8}+10 x_{1} x_{2}^{3}+60 \mu x_{1} x_{2}^{2} \\
& f_{2}^{\mathrm{B}}=0
\end{aligned}
$$




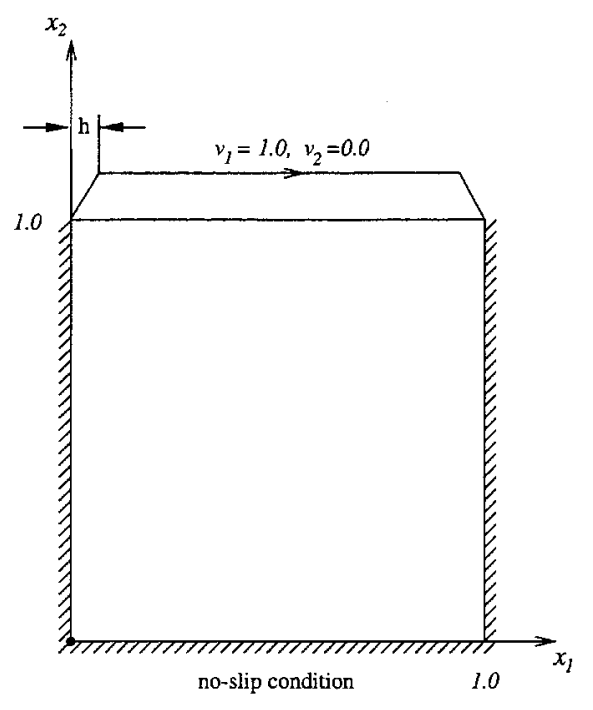

Figure 7. Driven flow cavity problem

and the fluid density is $\rho=1$. The exact solution for this problem is

$$
v_{1}=-5 x_{1} x_{2}^{4}, \quad v_{2}=-\frac{1}{2}+x_{2}^{5}, \quad p=\frac{1}{2} x_{2}^{5}-\frac{1}{2} x_{2}^{10}+5 \mu x_{2}^{4}
$$

Two cases are solved. In case 1 , we consider a diffusion dominated problem for which $\mu=10$. In this case, the Reynolds number is $R e=0.5025$, based on the maximum velocity and the length of the domain. In case 2 , we consider a pure convection problem with $\mu=0$. We perform the convergence study using $8 \times 8,16 \times 16$ and $32 \times 32$ uniform and distorted meshes, see Figure 3 for the coarsest meshes used. The finer meshes are obtained by simply subdividing the elements of the coarser mesh. The errors measured are: the error in the calculated pressure in the $L^{2}$-norm, $\left\|p-p_{h}\right\|_{L^{2}}$, and the velocity error using the $L^{2}$-norm, $\left\|\mathbf{v}-\mathbf{v}_{h}\right\|_{L^{2}}$. The solutions of the problem using the GLS method using the finest mesh are shown in Figure 4.

The results of the convergence study for case 1 are shown in Figure 5 and for case 2 in Figure 6.

For the diffusion dominated problem, the measured orders of convergence of all discretizations are the same and agree with a theoretical error analysis,

$$
\left\|p-p_{h}\right\|_{0} \sim c_{1} h^{2}
$$

and

$$
\left\|\mathbf{v}-\mathbf{v}_{h}\right\|_{0} \sim c_{2} h^{3}
$$

where $c_{1}$ and $c_{2}$ are constants. Futhermore, we notice that the convergence constants $c_{1}$ and $c_{2}$ are almost the same using the HDAD, SUPG and GLS procedures.

Note that using distorted element meshes does not change the order of convergence. However, the convergence constants in equations (8) and (9) are changed.

In the pure convection case, the numerical study shows that the orders of convergence of the velocity are less than in the diffusion-dominated case. The 9/4-c element discretization with 
Table I. Number of iterations required to solve the driven flow cavity problem with different Reynolds numbers using different upwind methods

\begin{tabular}{rcccc}
\hline \multicolumn{1}{l}{$R$} & HDAD & SUPG & S-SUPG & GLS \\
\hline 400 & 8 & 8 & 7 & 8 \\
1000 & 7 & 8 & 6 & 8 \\
2000 & 6 & 7 & 6 & 6 \\
3000 & 5 & 6 & 5 & 5 \\
4000 & 4 & $*$ & 5 & 6 \\
5000 & 4 & & 5 & 4 \\
6000 & 4 & & 4 & 4 \\
7000 & 4 & & 4 & 4 \\
8000 & 4 & & 4 & 4 \\
9000 & 5 & & 4 & 4 \\
10000 & $*$ & & 4 & 4 \\
11000 & & & 5 & 4 \\
12000 & & & 5 & 4 \\
13000 & & & 5 & 4 \\
14000 & & & 6 & 5 \\
15000 & & & 6 & 4 \\
16000 & & & 6 & 4 \\
18000 & & & 13 & 4 \\
20000 & & & $*$ & 4 \\
22000 & & & & 4 \\
24000 & & & & 4 \\
26000 & & & & \\
28000 & & & & \\
\hline
\end{tabular}

Note: Newton-Raphson method is used with convergence tolerance $=10^{-6}$; the $\left(^{*}\right)$ denotes iteration not converged

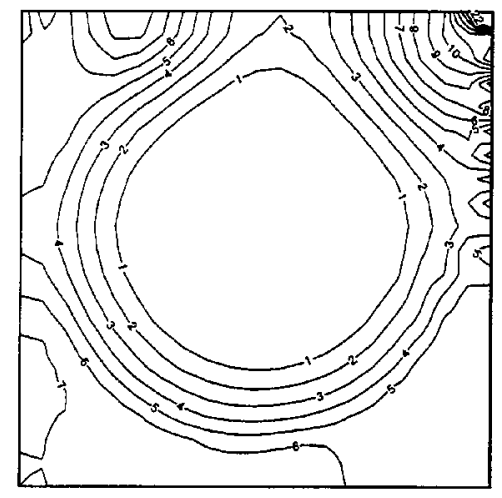

(a)

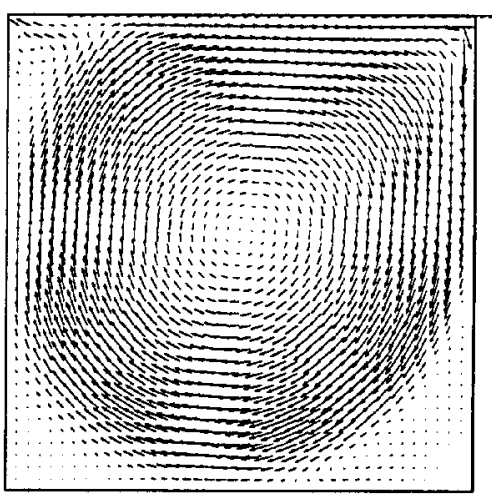

(b)

Figure 8. Solution of the driven flow cavity problem with $R e=5000$ using the $9 / 4-\mathrm{c}$ element with high-order derivative artificial diffusion method: (a) pressure contours; (b) velocity vectors 


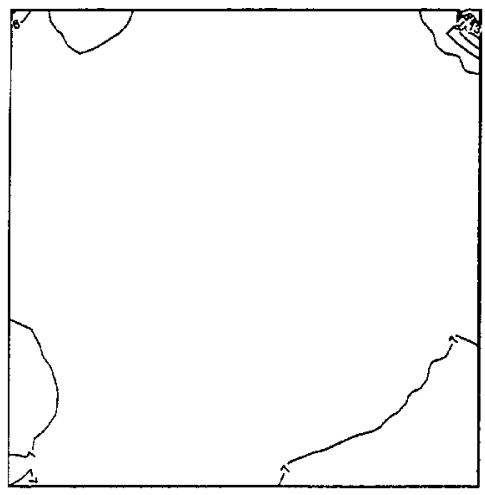

(a)
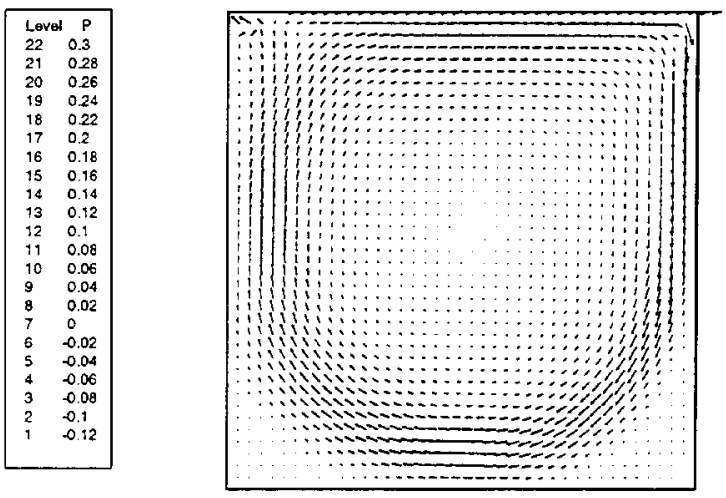

(b)

Figure 9. Solution of the driven flow cavity problem with $R e=5000$ using the 9/4-c element with simplified SUPG method: (a) pressure contours; (b) velocity vectors

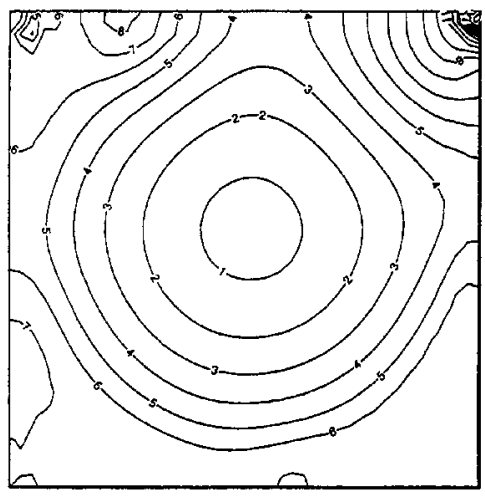

(a)

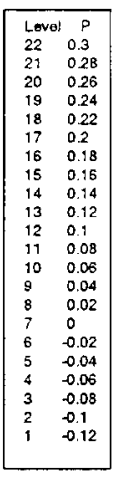

\begin{tabular}{ll}
22 & 0.3 \\
$0.2 B$ \\
0 & 0.26 \\
19 & 0.24 \\
8 & 0.22 \\
7 & 0.2 \\
6 & 0.18 \\
5 & 0.16 \\
4 & 0.14 \\
3 & 0.12 \\
2 & 0.1 \\
1 & 0.08 \\
0 & 0.06 \\
0.04 \\
0.02 \\
0 \\
-0.02 \\
-0.04 \\
-0.06 \\
-0.08 \\
-0.1 \\
-0.12 \\
\hline
\end{tabular}

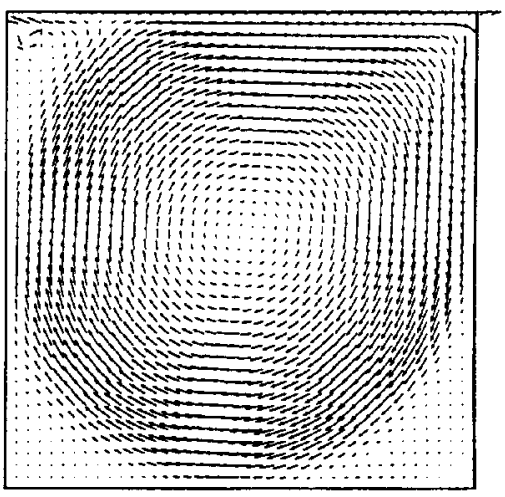

(b)

Figure 10. Solution of the driven flow cavity problem with $R e=5000$ using the 9/9-c element with GLS method: (a) pressure contours; (b) velocity vectors

the high-order derivative artificial diffusion method gives almost an order of convergence of 3 . The SUPG and GLS methods give almost the same orders of convergence, around 2, and the simplified SUPG method gives less than 2. Notice that, in this case, the use of distorted meshes results in better convergence rates for all methods because the mesh distortions favour the flow solution.

For the pressure variable, the discretizations using the HDAD, SUPG and GLS procedures give as good orders of convergence as in the diffusion-dominated problem, and in some cases even better values. Using the simplified SUPG method the order of convergence is, however, less than in the diffusion-dominated problem. 


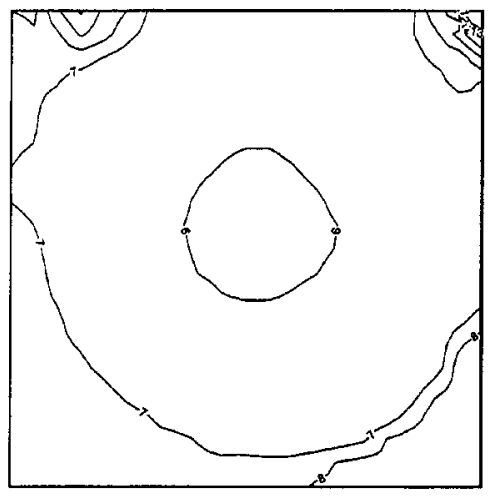

(a)

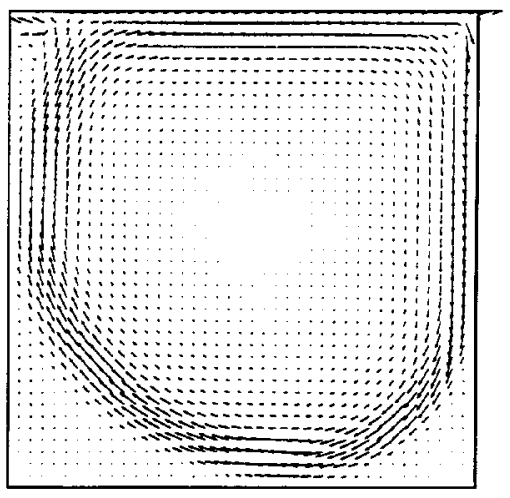

(b)

Figure 11. Solution of the driven flow cavity problem with $R e=15000$ using the $9 / 4-\mathrm{c}$ element with simplified SUPG method: (a) pressure contours; (b) velocity vectors

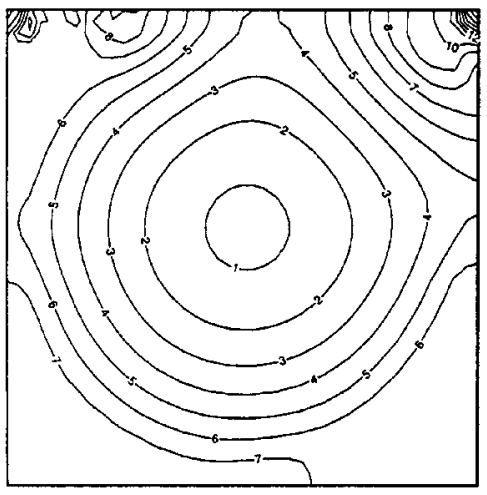

(a)
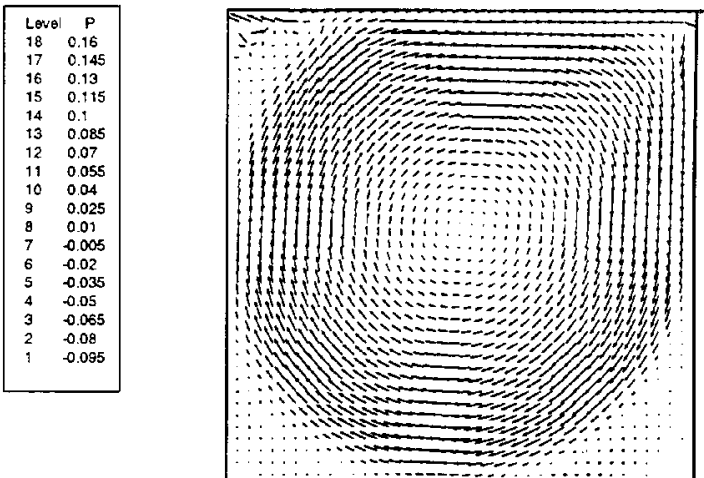

(b)

Figure 12. Solution of the driven flow cavity problem with $R e=15000$ using the 9/9-c element with GLS method: (a) pressure contours; (b) velocity vectors

\subsection{Driven flow cavity problem}

To compare the performance of the upwind methods described in the previous section in the solution of high Reynolds number flows, we consider the driven flow cavity problem. In comparing the methods, we increase the Reynolds number and focus on how high the Reynolds number can be before convergence is no longer reached in the allowed (35) number of iterations. We also measure how many iterations are required to solve the problem at different Reynolds numbers.

The problem considered is described in Figure 7. The no-slip boundary condition is imposed on the left, lower and right boundaries. On the upper boundary, we impose the condition $v_{1}=1, v_{2}=0$ distributed as shown in Figure 7. The domain is discretized into a uniform mesh of $20 \times 20$ elements. The Reynolds number of the problem is calculated based on the imposed velocity and the width of the domain. Zero pressure is prescribed at the lower left corner and a steady-state 


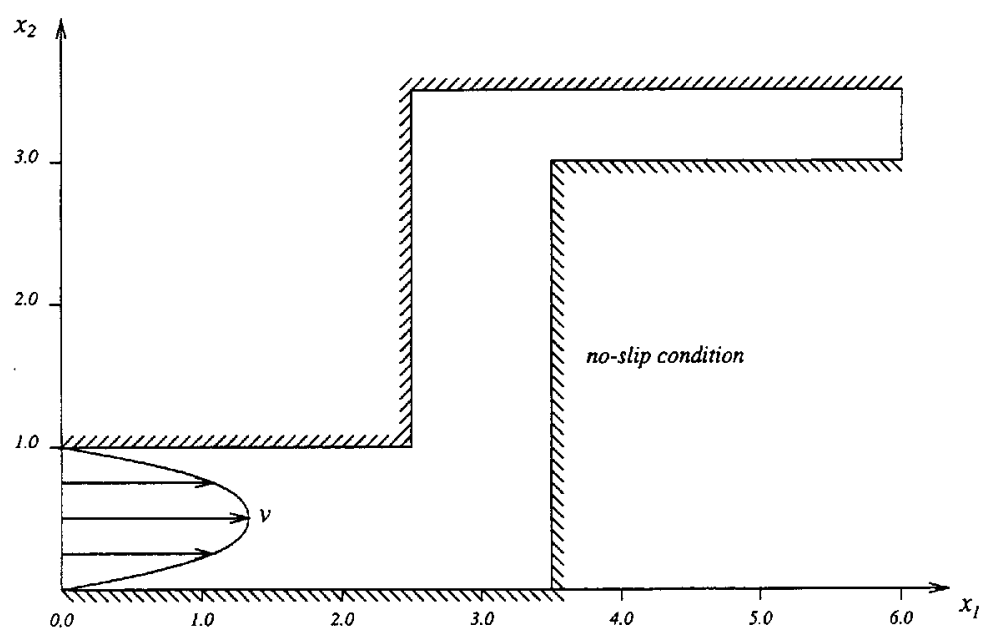

Figure 13. The 'S'-shaped channel flow problem

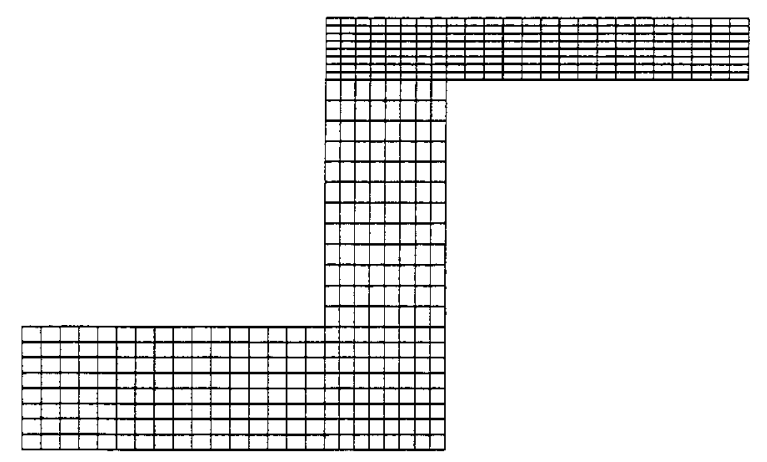

Figure 14. The mesh used for the 'S'-shaped channel flow problem

analysis is carried out. We perform runs with increasing Reynolds numbers as given in Table I and record the number of iterations. The Newton-Raphson method is used to solve the nonlinear equations with the convergence tolerance on the normalized norms of residuals in the velocities and pressure $\left(R_{v}=\left\|\Delta \mathbf{v}_{h}\left|/\left\|\mathbf{v}_{h}\right\|, R_{p}=\left\|\Delta p_{h} \mid /\right\| p_{h} \|\right)\right.\right.$ equal to $10^{-6}$. To reach the solutions for the Reynolds numbers listed in Table I, we start from zero pressure and velocities as initial condition, and use the converged solution of the lower Reynolds number case as the initial condition for the next higher Reynolds number problem.

The solutions of the problem for $R e=5000$ using different upwind methods are shown in Figures 8-10. In the solutions, the high-order derivative artificial diffusion method gives the largest pressure gradient and the GLS method gives the smallest pressure gradient. This is to be expected since the GLS method contains an artificial diffusion in the pressure term. Figure 8(a) shows that the HDAD method solution contains slight pressure oscillations. The velocity solution of the high-order derivative artificial diffusion method is similar to the solution of the GLS method. The simplified SUPG method gives a narrow banded velocity solution, see Figure 9(b). 
Table II. Number of iterations required to solve the ' $\mathrm{S}$ '-shaped channel problem with different Reynolds numbers using different upwind methods

\begin{tabular}{rcccc}
\hline \multicolumn{1}{l}{$R$} & HDAD & SUPG & S-SUPG & GLS \\
\hline 50 & 5 & 6 & 5 & 5 \\
250 & 5 & 8 & 5 & 5 \\
500 & 5 & $*$ & 5 & 6 \\
750 & 5 & & 5 & $*$ \\
1000 & $*$ & & 5 & \\
1500 & & & 5 & \\
2000 & & & 6 & \\
2500 & & & 6 & \\
3000 & & & 4 & \\
3500 & & & 5 & \\
4000 & & & 5 & \\
5000 & & & 9 & \\
6000 & & & 8 & \\
7000 & & & 16 & \\
8000 & & & 9 & \\
9000 & & & 6 & \\
10000 & & & 4 & \\
15000 & & & 7 & \\
20000 & & & & \\
\hline
\end{tabular}

Note: Newton-Raphson method is used with convergence tolerance $=10^{-6}$; the $\left(^{*}\right)$ denotes iteration not converged

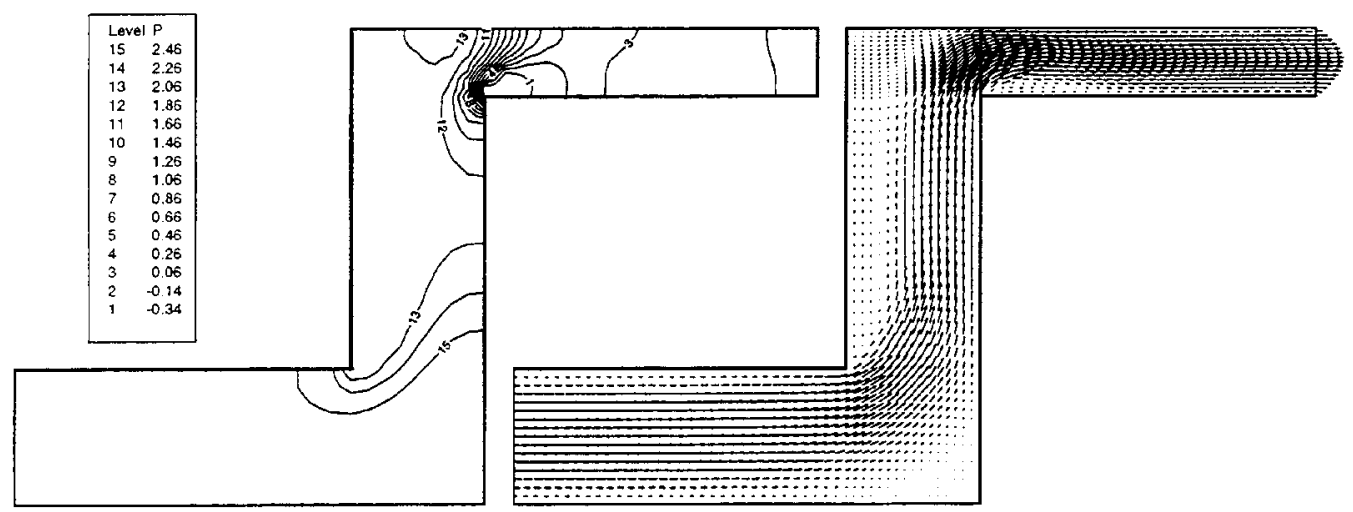

(a)

(b)

Figure 15. Solution of the "S"-shaped channel flow problem with $R e=250$ using the 9/4-c element with high-order derivative artificial diffusion method: (a) pressure contours; (b) velocity vectors

The solutions of the problem for $R e=15000$ using the simplified SUPG and GLS methods are shown in Figures 11 and 12. Note the significant difference in the solutions obtained. As we see from Table I, the GLS method is the last method to fail as the Reynolds number is increased. 


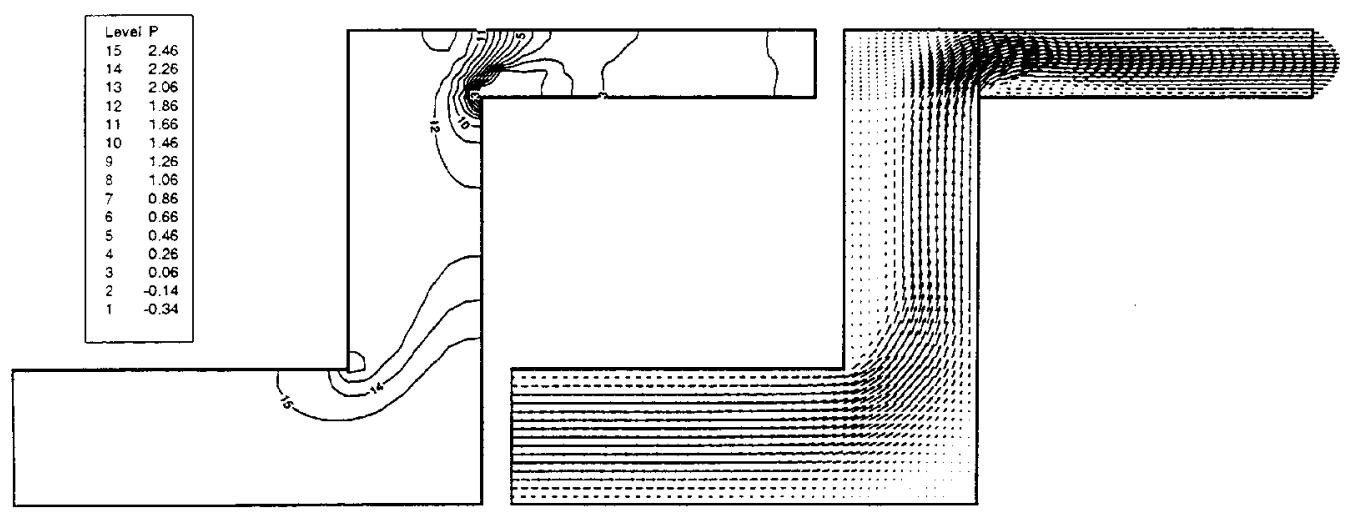

(a)

(b)

Figure 16. Solution of the "S"-shaped channel flow problem with $R e=250$ using the 9/4-c element with SUPG method: (a) pressure contours; (b) velocity vectors

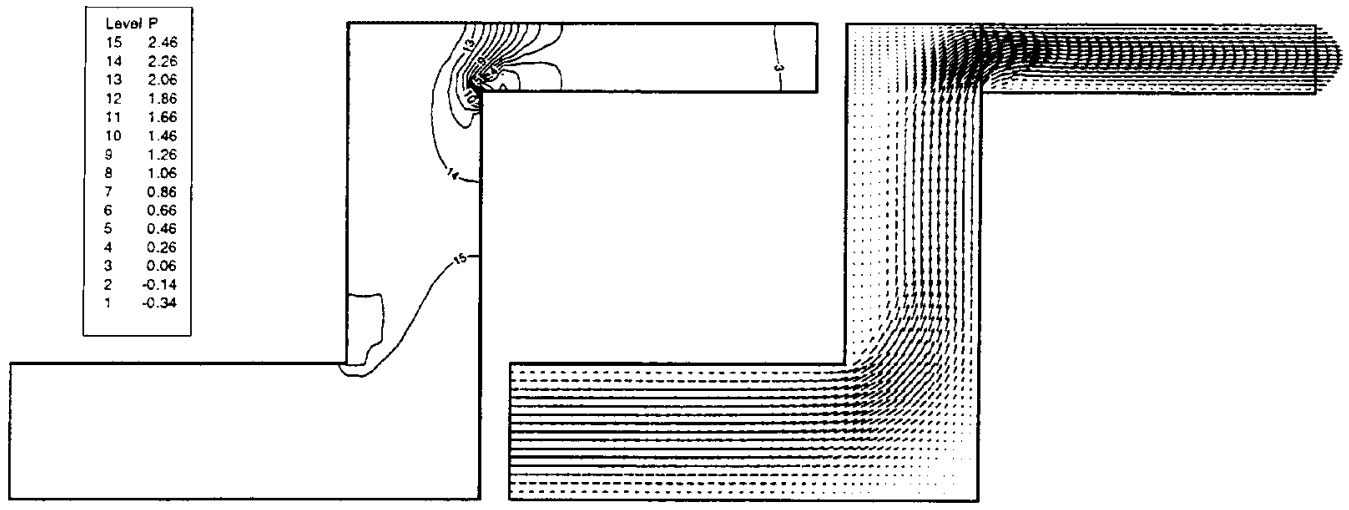

(a)

(b)

Figure 17. Solution of the "S"-shaped channel flow problem with $R e=250$ using the 9/4-c element with simplified SUPG method: (a) pressure contours; (b) velocity vectors

\subsection{S-shaped channel flow}

Like for the driven flow cavity problem, this problem is considered to study the performance of the upwind methods. We focus on establishing how high the Reynolds number of the problem can be for a solution still to be obtained, and how many iterations are required for the solution. The problem considered is described in Figure 13. The no-slip boundary condition is imposed on the walls of the channel. At the inlet, we impose the velocity with a parabolic profile and maximum unit velocity at the centre. At the outlet, zero tractions are imposed. The Reynolds number is 


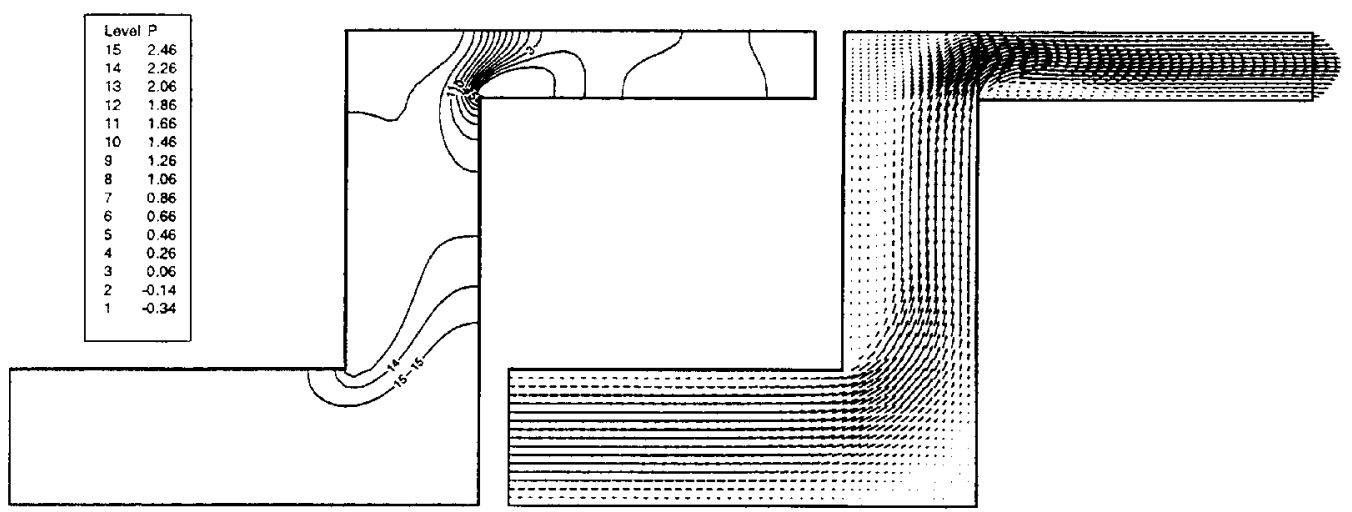

(a)

(b)

Figure 18. Solution of the "S"-shaped channel flow problem with $R e=250$ using the 9/9-c element with GLS method: (a) pressure contours; (b) velocity vectors

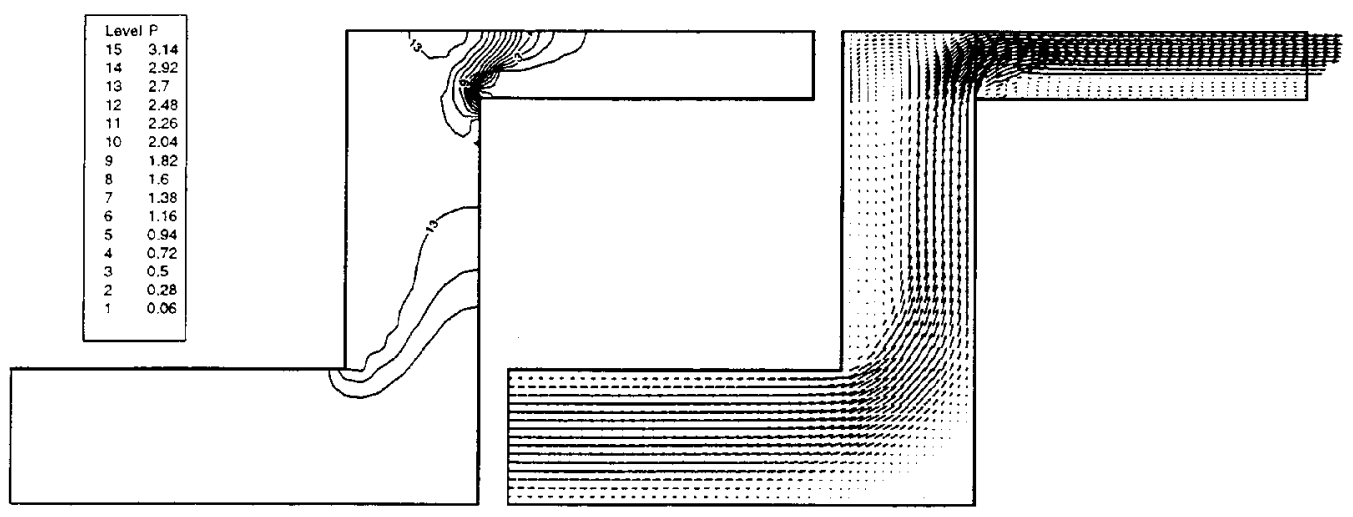

(a)

(b)

Figure 19. Solution of the "S"-shaped channel flow problem with $R e=15000$ using the 9/4-c element with simplified SUPG method: (a) pressure contours; (b) velocity vectors

calculated based on the maximum inlet velocity and the height of the channel at the inlet. The mesh used is shown in Figure 14.

A steady-state analysis is performed for each Reynolds number considered. The Reynolds number is increased as given in Table II. This table also gives the iterations required to solve the problem using the different upwind methods. The Newton-Raphson method is used to solve the nonlinear finite element equations and the convergence tolerance on the residual of normalized velocities and pressure is $10^{-6}$. Like for the driven flow cavity problem, in solving the problem with a certain Reynolds number, we use the solution of the lower Reynolds number as the initial condition. 


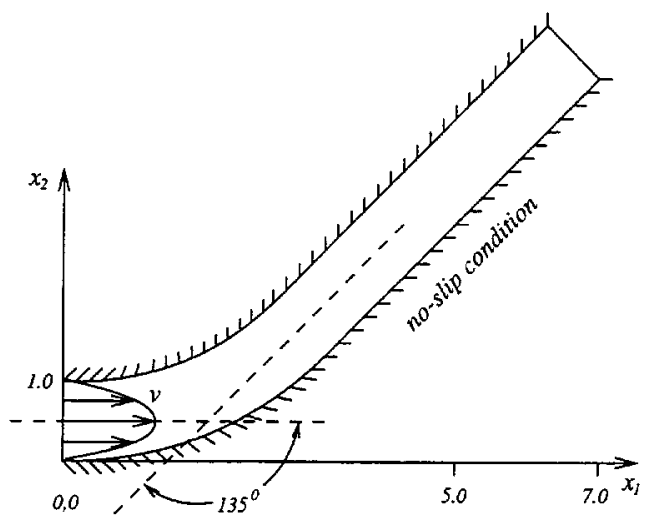

Figure 20. The curved-channel flow problem

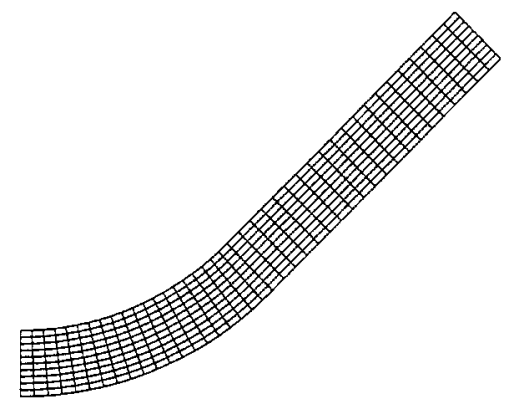

Figure 21. The mesh used for the curved-channel flow problem

Table III. Number of iterations required to solve the curved channel flow problem with different Reynolds numbers using different upwind methods

\begin{tabular}{rcccc}
\hline \multicolumn{1}{l}{$R e$} & HDAD & SUPG & S-SUPG & GLS \\
\hline 50 & 4 & 6 & 5 & 4 \\
500 & 4 & 4 & 4 & 4 \\
5000 & 4 & 5 & 4 & 5 \\
50000 & 4 & $*$ & $*$ & $*$ \\
500000 & 3 & & & \\
5000000 & 3 & & &
\end{tabular}

Note: The Newton-Raphson method is used with convergence tolerance $=10^{-6}$; the $(*)$ denotes iteration not converged

The solutions of the problem for $R e=250$ using different upwind methods are shown in Figures 15-18. Notice that all upwind methods give very similar pressure and velocity solutions.

The solutions of the problem for $R e=15000$ using the simplified SUPG method is shown in Figure 19. As we can see from Table II, the simplified SUPG method fails last as the Reynolds number is increased. 


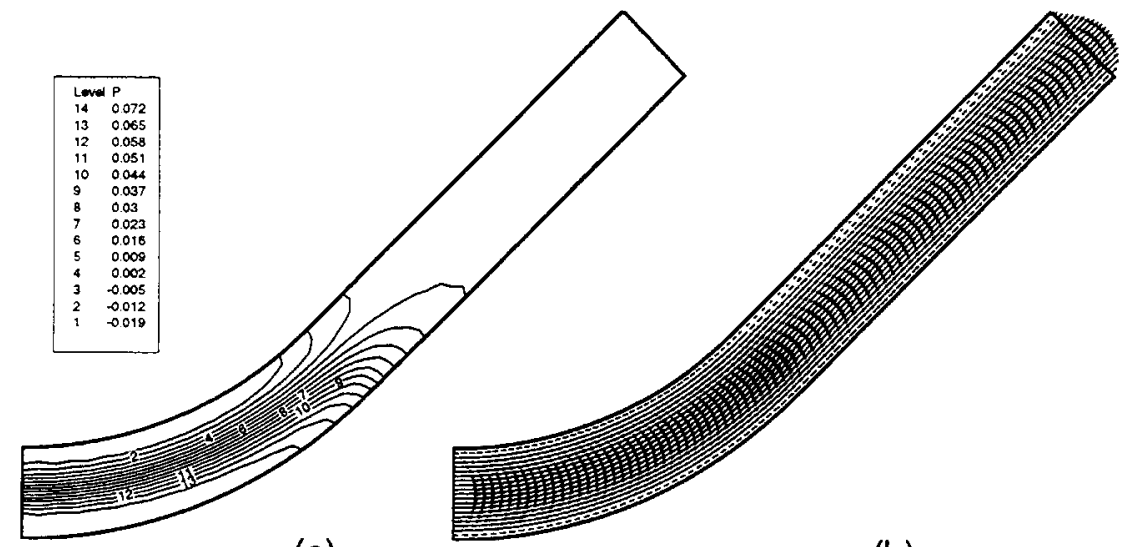

(a)

(b)

Figure 22. Solution of the curved channel flow problem with $R e=5000$ using the $9 / 4-c$ element with high-order derivative artificial diffusion method: (a) pressure contours; (b) velocity vectors

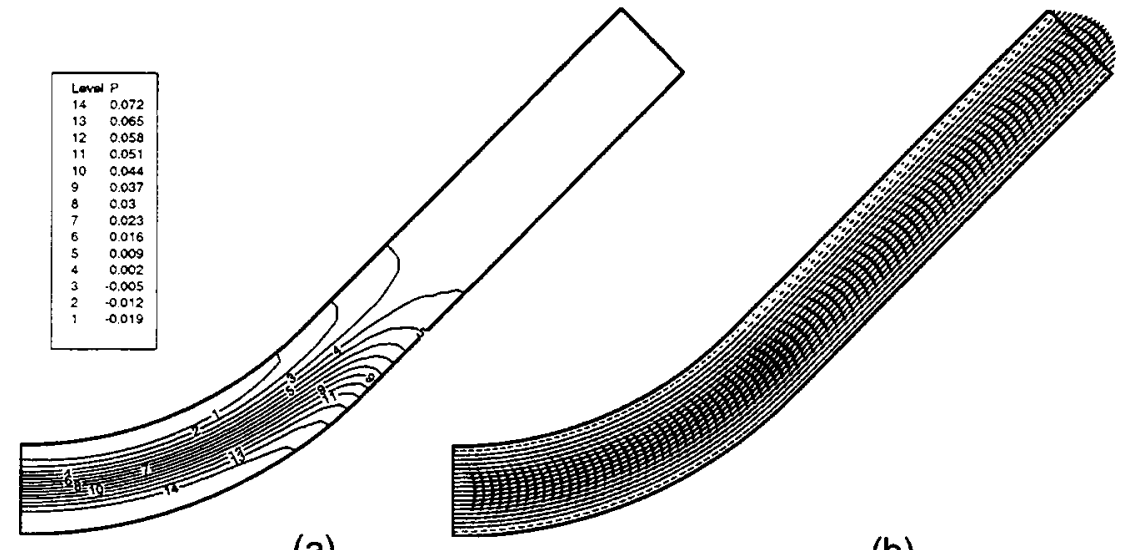

(a)

(b)

Figure 23. Solution of the curved channel flow problem with $R e=5000$ using the $9 / 4-\mathrm{c}$ element with SUPG method: (a) pressure contours; (b) velocity vectors

\subsection{Curved channel flow}

Unlike the other test problems, this problem does not contain any singularity. The problem considered is described in Figure 20. The no-slip condition is imposed on the upper and lower wall boundaries. At the inlet, we impose the velocity with a parabolic profile and the maximum velocity at the centre is unity. At the outlet, we impose zero tractions. The Reynolds number is calculated based on the maximum inlet velocity and the height of the channel at the inlet. The mesh used is shown in Figure 21. 


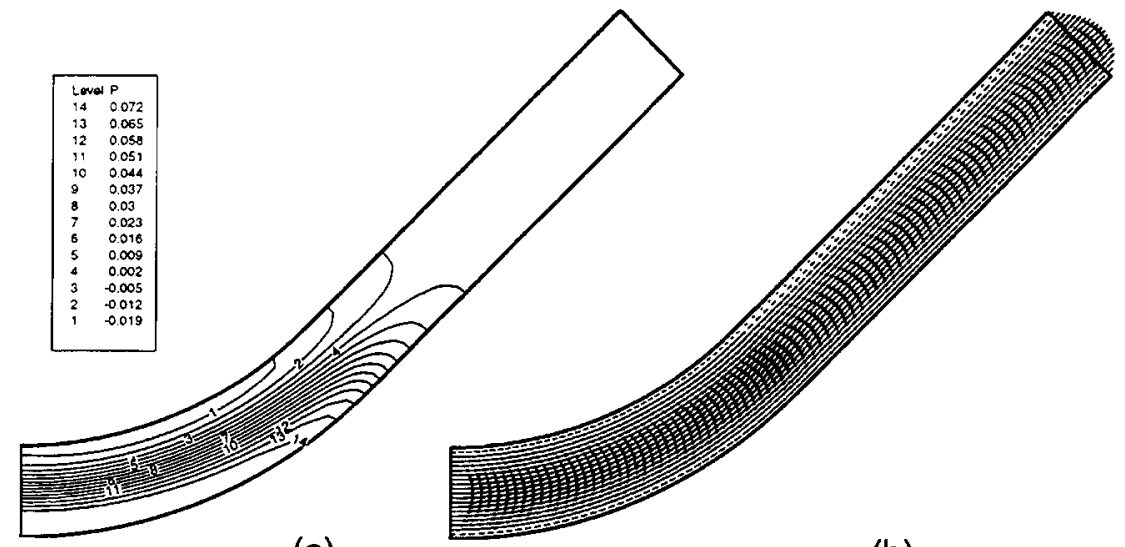

(a)

(b)

Figure 24. Solution of the curved channel flow problem with $R e=5000$ using the $9 / 4-c$ element with simplified SUPG method: (a) pressure contours; (b) velocity vectors

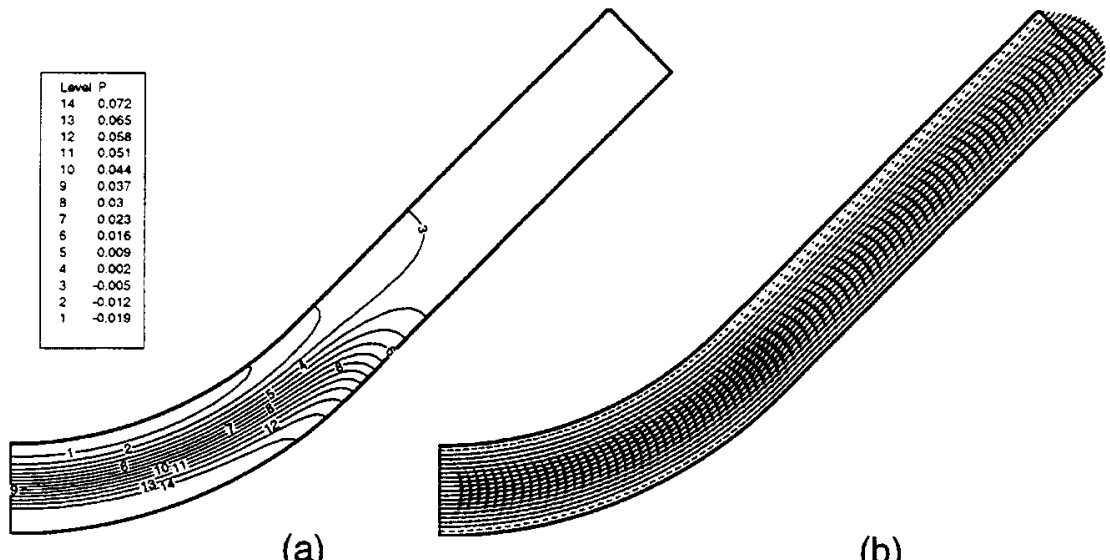

(b)

Figure 25. Solution of the curved channel flow problem with $R e=5000$ using the $9 / 9-\mathrm{c}$ element with GLS method: (a) pressure contours; (b) velocity vectors

We solve for the steady-state response and increase the Reynolds number as given in Table III, and record the number of iterations.

Like for the other problems, the Newton-Raphson method is used to solve the nonlinear equations with the convergence tolerance on the velocities and pressure equal to $10^{-6}$. To reach the solutions for the Reynolds numbers listed in Table III, we start from zero pressure and velocities as initial condition and use the converged solution of the lower Reynolds number case as the initial condition for the next higher Reynolds number problem.

The solutions of the problem with $R e=5000$ using different upwind methods are shown in Figures 22-25. Note that all upwind methods give very similar pressure and velocity solutions.

The solution of the problem for the Reynolds number 5000000 using the high-order derivative artificial diffusion method is shown in Figure 26. As we see from Table III, the high-order 


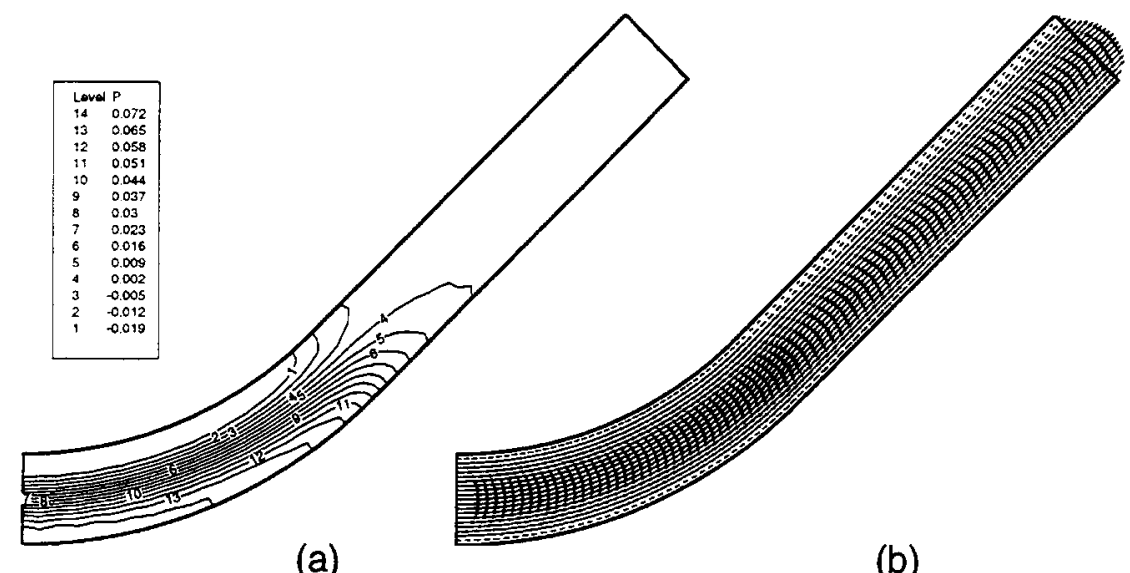

(a)

(b)

Figure 26. Solution of the curved channel flow problem with $R e=5000000$ using the 9/4-c element with high-order derivative artificial diffusion method: (a) pressure contours; (b) velocity vectors

derivative artificial diffusion method is the only method to solve the problem when the Reynolds number is very large.

\section{CONCLUSIONS}

The aim in this study was to evaluate some upwind techniques when used in parabolic finite element discretizations. We first described the characteristics of an 'ideal' upwind procedure and then reviewed and evaluated four upwind techniques: the high-order derivative artificial diffusion (HDAD) method, the SUPG technique, a simplification thereof, and the GLS method.

All methods performed quite well in a convergence study using a simple problem for which the exact solution is available. In the diffusion-dominated case, the $L_{2}$-norm orders of convergence in pressure and velocity were two and three, respectively. In the convection dominated case, the $L_{2}$-norm order of convergence in pressure remained two (except using the simplified SUPG method), whereas the velocity order of convergence decreased with different amounts for the methods to values between two and three. Of course, different convergence constants were measured for the techniques.

Three other test problems were solved. In each case the Reynolds number was increased in steps and we measured how many iterations were required for solution at a given Reynolds number and how high the Reynolds number could be for solution. The methods performed differently in the problem solutions with no method clearly outperforming the others. None of the methods tested could solve all high Reynolds number problems with the meshes used and hence no method showed characteristics close to the 'ideal' solution scheme. Indeed, the measured solution characteristics were rather far from the desired qualities which underlines the need for further major advances in the field.

\section{REFERENCES}

1. Brezzi F, Fortin M. Mixed and Hybrid Finite Element Methods. Springer: Berlin, 1991.

2. Bathe KJ. Finite Element Procedures. Prentice Hall: New Jersey, 1996. 
3. Brezzi F, Pitkäranta J. On the stabilization of finite element approximations of the Stokes equations. In Efficient Solutions of Elliptic Systems: Proceedings of a GAMM-Seminar, Notes on Numerical Fluid Mechanics, vol. 10, Hackbush W (ed.). Braunschweig, 1984.

4. Tezduyar TE, Mittal S, Ray SE, Shih R. Incompressible flow computations with stabilized bilinear and linear equalorder-interpolation velocity-pressure elements. Computer Methods in Applied Mechanics and Engineering 1992; 95:221-242.

5. Storti M, Nigro N, Idelsohn S. Equal-order interpolations: a unified approach to stabilize the incompressible and advective effects. Computer Methods in Applied Mechanics and Engineering 1997; 143:317-331.

6. Codina R, Blasco J. A finite element formulation for the Stokes problem allowing equal velocity-pressure interpolation. Computer Methods in Applied Mechanics and Engineering 1997; 143:373-391.

7. Brooks AN, Hughes TJR. Streamline upwind/Petrov-Galerkin formulations for convection dominated flows with particular emphasis on the incompressible Navier-Stokes equations. Computer Methods in Applied Mechanics and Engineering 1982; 32:199-259.

8. Hughes TJR, Mallet M, Mizukami A. A new finite element formulation for computational fluid dynamics: II. Beyond SUPG. Computer Methods in Applied Mechanics and Engineering 1986; 54:341-355.

9. Hughes TJR, Franca LP, Hulbert GM. A new finite element formulation for computational fluid dynamics: VIII. The Galerkin/Least-squares method for advective-diffusive equations. Computer Methods in Applied Mechanics and Engineering 1989; 73:173-189.

10. Brezzi F, Bristeau MO, Franca LP, Mallet M, Roge G. A relationship between stabilized finite element methods and the Galerkin method with bubble functions. Computer Methods in Applied Mechanics and Engineering 1992; 96:117-129.

11. Brezzi F, Marini D, Russo A. Applications of the pseudo residual-free bubbles to the stabilization of convection-diffusion problems. Computer Methods in Applied Mechanics and Engineering 1998; 166:51-63.

12. Donea J, Belytschko T, Smolinski P. A generalized Galerkin method for steady convection-diffusion problems with application to quadratic shape function elements. Computer Methods in Applied Mechanics and Engineering 1985; 48:25-43.

13. Demkowicz L, Oden JT, Rachowicz W, Hardy O. An h-p Taylor-Galerkin finite element method for compressible Euler equations. Computer Methods in Applied Mechanics and Engineering 1991; 88:363-396.

14. Hendriana D, Bathe KJ. On a parabolic quadrilateral finite element for compressible flows. Computer Methods in Applied Mechanics and Engineering, in press.

15. Hannani SK, Stanislas M, Dupont P. Incompressible Navier-Stokes computations with SUPG and GLS formulations -A comparison study. Computer Methods in Applied Mechanics and Engineering 1995; 124:153-170.

16. Codina R, Onate E, Cervera M. The intrinsic time for the streamline upwind/Petrov-Galerkin formulation using quadratic elements. Computer Methods in Applied Mechanics and Engineering 1992; 94:239-262.

17. Franca LP, Frey SL. Stabilized finite element methods: II. The incompressible Navier-Stokes equations. Computer Methods in Applied Mechanics and Engineering 1992; 99:209-233. 